\title{
Efficient Abstraction Refinement in Interpolation-Based Unbounded Model Checking ${ }^{\star}$
}

\author{
Bing Li and Fabio Somenzi \\ University of Colorado at Boulder \\ \{Bing.Li, Fabio\}@Colorado.EDU
}

\begin{abstract}
It has been pointed out by McMillan that modern satisfiability (SAT) solvers have the ability to perform on-the-fly model abstraction when examining it for the existence of paths satisfying certain conditions. The issue has therefore been raised of whether explicit abstraction refinement schemes still have a role to play in SAT-based model checking. Recent work by Gupta and Strichman has addressed this issue for bounded model checking (BMC), while in this paper we consider unbounded model checking based on interpolation. We show that for passing properties abstraction refinement leads to proofs that often require examination of shorter paths. On the other hand, there is significant overhead involved in computing efficient abstractions. We describe the techniques we have developed to minimize such overhead to the point that even for failing properties the abstraction refinement scheme remains competitive.
\end{abstract}

\section{Introduction}

Model checking algorithms that employ propositional satisfiability (SAT) as basic decision procedure have enjoyed considerable success in the last few years. Bounded Model Checking (BMC, [2]) can often find counterexamples to properties of models that are too complex for other techniques. In BMC the problem of finding a trace violating a linear-time property is formulated as the satisfiability of a formula derived from the transition relation of the model, the initial state condition, and the property to be disproved. The practical success of this approach is due in part to the great efficiency achieved by today's SAT solvers, and in part to the fact that storing a representation of the set of reachable states of the model is not required.

Although theoretically complete for finite-state systems, the basic BMC algorithm is not useful to prove properties (rather than refuting them). Auxiliary techniques have been developed to provide termination conditions for true properties. The method of [17] checks for simple paths of certain lengths that either start from initial states or end in bad states. From the non-existence of such paths it is possible to deduce the non-reachability of the bad states, and hence to prove an invariant. A criterion for all linear-time properties also relying on simple paths was proposed in [1]; it is based on representing the property as a Büchi automaton.

A different approach to proving termination is taken in [15], which relies on the notion of Craig's interpolant of an unsatisfiability proof. A properly chosen interpolant of the proof that no counterexample of length $L$ to an invariant exists can be interpreted as

\footnotetext{
* This work was supported in part by SRC contract 2004-TJ-920.
} 
a set of states with two properties: It includes all states reachable in one transition from the initial states; and it contains no state that can reach a state violating the invariant in $L-1$ transitions or less. This observation suggests an iterative procedure which, if it converges without discovering any (possibly spurious) counterexample, guarantees the unreachability of the bad states. The advantage of interpolation-based termination check is that the paths to be encoded in the SAT instances are never longer than those examined by the simple-path method and sometimes much shorter. Both approaches turn bounded model checking algorithms into unbounded ones. There also exist SATbased algorithms that are not extensions of BMC (e.g., [14]) but so far they have proved competitive only for a limited class of problems.

Abstraction refinement [10] is another technique that has greatly improved the capacity of model checkers. To prove a (universal) linear-time property, one starts with a coarse abstraction that simulates the given model. If no counterexample is found in the abstract model, the property is known to hold in the original, concrete system as well. If a counterexample is found, which is not also a counterexample of the concrete model, the abstraction is too coarse and is refined. Refinement may aim at removing the error trace that was discovered [5], or all error traces of a certain length [16, 4, 20, 12].

Whether SAT-based model checking and abstraction refinement can be profitably combined is an interesting question. It has been argued convincingly by McMillan that modern SAT solvers effectively perform an on-the-fly abstraction of the model. They can prove unsatisfiability of a formula without ever assigning values to many of its variables. The heuristics used to choose decision variables indeed tend to identify a good subset of the variables and mostly work on them. With abstraction already taking place inside the SAT solver, it is not clear that abstraction outside the solver would provide additional benefit. On the other hand, there may be significant overhead in checking spurious counterexamples and computing the corresponding refinements.

Gupta and Strichman [8] have addressed this issue for BMC, that is, for failing properties. They observed that SAT solvers may spend time on needless Boolean constraint propagation and local conflicts if working directly on a large concrete model.

In this paper we look at the combination of abstraction refinement and interpolationbased model checking. We show that termination test carried out on the abstract model increases the ability to prove properties for large models by reducing the length of the paths to be examined. This improvement depends on the model checker ability to keep the abstract model small. Therefore, our algorithm invests considerable time in identifying small refinements. This, however, adds a significant overhead in the case of failing properties, for which the ability to prove termination sooner is immaterial. Therefore, we present several techniques that we have devised to speed up the computation of refinements without compromising their quality. The resulting algorithm outperforms the corresponding one without abstraction refinement for passing properties, while remaining competitive for the failing ones.

The rest of the paper is organized as follows. Section 2 reviews background material. Sections 3 and 4 present the abstraction refinement algorithm with termination check based on interpolation. Experimental results are discussed in Sect. 5 and conclusions are drawn in Sect. 6 . 


\section{Preliminaries}

\subsection{Open Systems and Safety Properties}

Let $V=\left\{v_{1}, \ldots, v_{n}\right\}$ and $W=\left\{w_{1}, \ldots, w_{m}\right\}$ be sets of Boolean variables. We designate by $V^{\prime}$ the set $\left\{v_{1}^{\prime}, \ldots, v_{n}^{\prime}\right\}$ consisting of the primed version of the elements of $V$, and by $V^{i}$ the set $\left\{v_{1}^{i}, \ldots, v_{n}^{i}\right\}$. Likewise, $W^{i}=\left\{w_{1}^{i}, \ldots, w_{m}^{i}\right\}$. An open system is a 4-tuple $\langle V, W, I, T\rangle$, where $V$ is the set of (current) state variables, $W$ is the set of combinational variables, $I(V)$ is the initial state predicate, and $T\left(V, W, V^{\prime}\right)$ is the transition relation. The variables in $V^{\prime}$ are the next state variables. We assume that $T\left(V, W, V^{\prime}\right)$ is given by a circuit graph, that is, by a labeled graph $\mathcal{C}=(V \cup W, E)$ such that $m \geq n$, node $v_{i} \in V$ is labeled by $w_{i} \in W$, node $w_{i} \in W$ is labeled by a Boolean formula $T_{i}=w_{i} \leftrightarrow \delta_{i}(V, W),\left(w_{i}, v_{i}\right) \in E$ for $i \in\{1, \ldots, n\}$, and, for $x \in V \cup W, w_{i} \in W,\left(x, w_{i}\right) \in E$ iff $x$ appears in $\delta_{i}$. The transition relation is then defined by:

$$
T\left(V, W, V^{\prime}\right)=\bigwedge_{1 \leq i \leq n}\left(v_{i}^{\prime} \leftrightarrow w_{i}\right) \wedge \bigwedge_{1 \leq i \leq m} T_{i}(W, V)
$$

In this paper, we use a form of circuit graph known as And-Inverter Graph [9] from the only types of gates allowed in it. AIGs can be manipulated efficiently.

An open system $\Omega$ defines a labeled transition structure in the usual way, with states $Q_{\Omega}$ corresponding to the valuations of the variables in $V$, and transition labels corresponding to the valuations of the variables in $W$. Conversely, a set of states $S \subseteq Q_{\Omega}$ corresponds to a predicate $S(V)$ or $S\left(V^{\prime}\right)$. Predicate $S(V)\left(S\left(V^{\prime}\right)\right)$ is the characteristic function of $S$ expressed in terms of the current (next) state variables. State $q \in Q_{\Omega}$ is an initial state if it satisfies $I(V)$. State set $S \subseteq Q_{\Omega}$ is reachable from state set $S^{\prime}$ in $k$ steps if there is a path of length $k$ in the labeled transition structure defined by $\Omega$ that connects some state in $S^{\prime}$ to some state in $S$; equivalently if

$$
S^{\prime}\left(V^{0}\right) \wedge \bigwedge_{1 \leq i \leq k} T\left(V^{i-1}, W^{i}, V^{i}\right) \wedge S\left(V^{k}\right)
$$

is satisfiable. State set $S$ is reachable from $S^{\prime}$ if there exists $k \in \mathbb{N}$ such that $S$ is reachable in $k$ steps from $S^{\prime}$. A state set is reachable (in $k$ steps) if it is reachable (in $k$ steps) from $I$. A sequence of states $\rho \in Q_{\Omega}^{*}\left(\in Q_{\Omega}^{\omega}\right)$ is a run of $\Omega$ if the first state is initial, and every other state is reachable from its predecessor in one step.

An invariant is a property that states that a certain predicate holds of all reachable states of $\Omega$. Let $P$ be the set of states that satisfy that predicate. We identify the property with the set of states that satisfy it. Hence, property $P$ is satisfied by $\Omega$ if there is no $k \in \mathbb{N}$ such that

$$
I\left(V^{0}\right) \wedge \bigwedge_{1 \leq i \leq k} T\left(V^{i-1}, W^{i}, V^{i}\right) \wedge \neg P\left(V^{k}\right)
$$

is satisfiable. This approach extends to all safety properties. (See, for instance, [12].)

The search for a $k$ such that $(3)$ is satisfiable can obviously be restricted to the range $\left\{0, \ldots,\left|Q_{\Omega}\right|-1\right\}$. Hence, in theory, the process is guaranteed to terminate. In practice, the number of states is too large to be of any practical use, and tighter upper bounds for $k$ are sought [17, 15]. 


\subsection{Abstraction Refinement}

Open system $\widehat{\Omega}=\langle\widehat{V}, \widehat{W}, \widehat{I}, \widehat{T}\rangle$ is an abstraction of $\Omega$ if

$-\widehat{V} \subseteq V$

- $\widehat{W}^{-}=\widehat{W}_{1} \cup(V \backslash \widehat{V})$;

- $\widehat{W}_{1} \subseteq W$ such that $v_{i} \in \widehat{V}$ implies $w_{i} \in \widehat{W}_{1}$;

- $\widehat{I}(\widehat{V})=\exists(V \backslash \widehat{V}) \cdot I(V)$;

- $\widehat{T}\left(\widehat{V}, \widehat{W}, \widehat{V}^{\prime}\right)=\exists(W \backslash \widehat{W}) . \exists\left(V^{\prime} \backslash \widehat{V}^{\prime}\right) . T\left(V, W, V^{\prime}\right)$.

(Note that $w_{i}$ is the combinational variable associated to $v_{i}^{\prime}$ ). This definition entails that every run of $\Omega$ has a matching run in $\widehat{\Omega}$. Property $\widehat{P}$ is the abstraction of property $P$ with respect to $\widehat{\Omega}$ if $\widehat{P}(\widehat{V})=\forall(V \backslash \widehat{V}) . P(V)$. If $\widehat{\Omega}$ satisfies (or models) $\widehat{P}$, then $\Omega$ satisfies $P$. That is, $\widehat{\Omega} \models \widehat{P} \rightarrow \Omega \models P$. This preservation result is the basis for the following abstraction refinement approach to the verification of $P$. One starts with a coarse abstraction $\widehat{\Omega}_{0}$ of the concrete open system $\Omega$ and checks whether $\widehat{\Omega}_{0} \models \widehat{P}_{0}$. If that is the case, then $\Omega \models P$; otherwise, there exists a least $k^{\prime} \in \mathbb{N}$ such that

$$
\widehat{I}\left(\widehat{V}^{0}\right) \wedge \bigwedge_{1 \leq i \leq k^{\prime}} T\left(\widehat{V}^{i-1}, \widehat{W}^{i}, \widehat{V}^{i}\right) \wedge \neg \widehat{P}\left(\widehat{V}^{k^{\prime}}\right)
$$

is satisfiable. The satisfying assignments to (4) are the shortest-length abstract counterexamples (ACEs). If $\widehat{\Omega}_{0} \forall \widehat{P}_{0}$ one or more ACEs are checked for concretization. That is, one checks whether (3) has solutions that agree with the ACE(s) being checked. Because of the additional constraints provided by the ACEs, a concretization test is often less expensive that the satisfiability check of (3). However, its failure only indicates that the abstract error traces are spurious. Therefore, if the concretization test fails, one chooses a refined abstraction $\widehat{\Omega}_{1}$ and repeats the process, until one of these cases occurs.

1. $\widehat{\Omega}_{i} \models \widehat{P}_{i}$ for some $i$, in which case $\Omega \models P$ is inferred.

2. The concretization test passes for some $i$, in which case it is concluded that $\Omega \not \models P$ and the satisfying assignment to (3) found is returned as counterexample to $P$.

3. The refinement eventually produces $\widehat{\Omega}_{i}=\Omega$. In this final case, the satisfiability check of (4) answers the model checking question conclusively.

The cone of influence (COI), or direct support, of a property is the union of the COIs (direct supports) of all the variables mentioned in the predicate $P$ that defines the property. COI reduction refers to the abstraction in which $\widehat{V}$ is the COI of the property. It is commonly applied before any model checking is attempted, because it satisfies

$$
\widehat{\Omega} \models \widehat{P} \leftrightarrow \Omega \models P .
$$

\subsection{Satisfiability Solvers, Proofs of Unsatisfiability, and Interpolants}

The Davis-Putnam-Logemann-Loveland (DPLL) procedure is the basic algorithm for most modern SAT solvers, which add conflict-driven learning and non-chronological backtracking [18] to the basic branching and backtracking approach. 
Conflict-driven learning is an important feature of many modern SAT solvers, which is normally based on conflict clause recording. Whenever SAT solvers detect a conflicting assignment to a formula $f$ (one that causes $f$ to evaluate to false), they conjoin a conflict clause to $f$. The clause is generated by analyzing the so-called implication graph, which shows which decisions and clauses are responsible for the conflict. The new clause prevents the solver from attempting the same assignment again. It may also exclude from future consideration other parts of the search space that can be inferred to contain no satisfying assignments.

The clauses that make up the edges of an implication graph can be used to explain the conflict clause deduced from it. Keeping track of the explanations of conflict clauses results in the ability to identify an unsatisfiable core (or unsatisfiability proof) [7, 21] of the given formula when it is indeed unsatisfiable. This can be done by recursively replacing conflict clauses with those that produced them, the resolvent of which is exactly the conflict clause. The process starts from the final empty clause (final unsolvable conflict) and terminates when only clauses of the original formula are left.

When the check for existence of counterexamples of a certain length fails, the unsatisfiable core produced by the SAT solver can be used to guide the refinement of the abstract model. AROSAT [11] is a specialized SAT solver that tries to find an unsatisfiability proof that relies on a small number of state variables of the model, so as to speed up the computation of a minimal refinement.

From the proof of unsatisfiability it is also possible to extract interpolants, that is, formulae that summarize part of the proof, while depending only on a specified set of variables [15]. Interpolants can be computed in time linear in the size of the resolution tree associated with the unsatisfiability proof.

\section{Algorithm}

Our algorithm is called IPAR because it combines interpolation and abstraction refinement. The pseudocode of our main procedure is shown in Fig. 1, The input to the algorithm is an open system $\Omega=\langle V, W, I, T\rangle$ whose transition relation $T$ is specified by a circuit graph $\mathcal{C}=(V \cup W, E)$, and a predicate $P(V)$ describing a set of accepting states. The return value is TRUE if the property passes, FALSE otherwise.

Initially, an abstract model $\widehat{\Omega}$ is computed by collecting in $\widehat{V}$ only the state variables that appear in $P(V)$; hence, $\widehat{P}=P$ throughout. Then the procedure of GetRefineMENTFROMREFPREDICT is applied to get probable future refinement and add it to $\widehat{\Omega}$. After the initial abstract model is created, the algorithm progressively increases $L$ from its initial value 0 until either a counterexample of length $L$ is found in the concrete system $\Omega$, or it is concluded that no counterexample exists in the current abstract model $\widehat{\Omega}$. For each abstract model, the procedure CHECKINTERPOLATION is invoked to detect the existence of counterexamples as well as prove absence of counterexamples by detecting convergence of the interpolants, which is similar to what is described in [15], with the differences that 1) the constraint due to the property is added only to the last timeframe, 2) each time the length is increased, its new value is the sum of the previous length and the number of iterations in the previous termination check minus one [13].

CHECKInTERPOLATION returns two parameters. The first is CEXFound; if it is FALSE, there is no counterexample of any length in the abstract model, and the prop- 


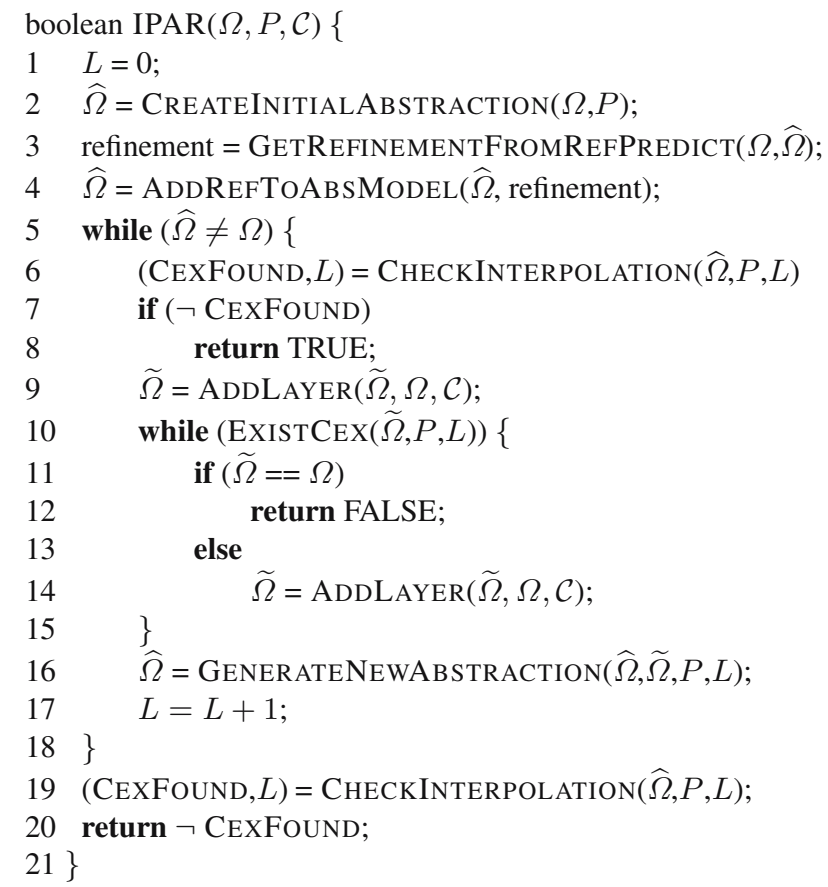

Fig. 1. The IPAR algorithm

erty is true of the concrete model. Otherwise, an abstract counterexample has been found, and we should try to find a corresponding real counterexample. The other parameter is $L$; if CEXFOUND is TRUE, there is no counterexample of length $<L$, but at least one counterexample of length $L$ in the abstract model. If CHECKINTERPOLATION fails to prove termination, we try to find a sufficient model-one that has no counterexample up to length $L$ - by incrementally adding, with ADDLAYER, latches to the current abstract model one layer at the time, until we either find a sufficient model or a real counterexample. Function EXISTCEX decides whether a counterexample exists by calling the SAT solver. Lines 9-15 in Fig. 1 implement a procedure called incremental concretization; more details can be found in [12]. Line 16 shows that if a sufficient model is found, a refinement is computed and added to the abstract model; the detailed pseudocode is shown in Fig. 2 Once a new abstract model is generated, we increase the length $L$ by one and iterate. The abstract model may eventually equal the concrete model; in this case we just apply the interpolation algorithm to the concrete model, as shown in Lines 19-20.

The pseudocode of GenERATENEwABSTRACTION is shown in Fig. 2 It takes four input parameters - concrete model $\Omega$, abstract model $\widehat{\Omega}$, property $P$, current checking length $L$-and returns a new abstract model. In this procedure, first a compact unsatisfiability proof is generated in procedure GENERATEUNSATPROOFFROMIAROSAT by utilizing IAROSAT (see Sect. 3.1); then bridge abstraction is applied to extract a sufficient refinement candidate set which, if added to $\widehat{\Omega}$, would kill all abstract counterexamples of length up to $L$. All latch variables (variables corresponding to some 


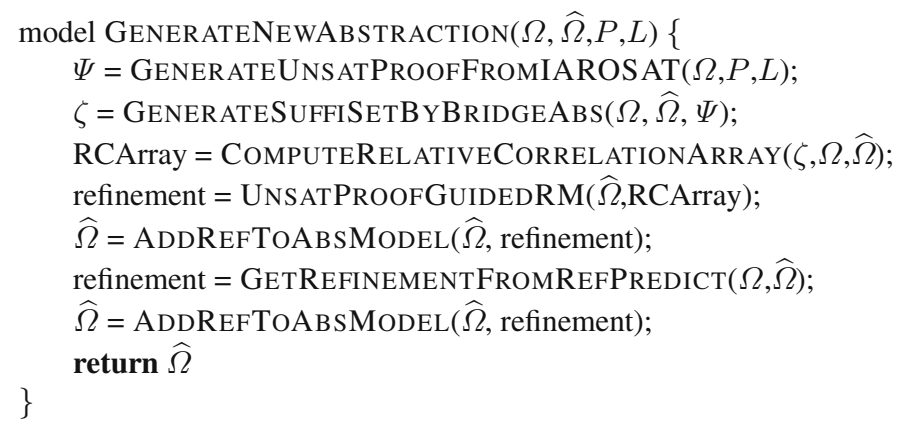

Fig. 2. The refinement algorithm

latch in the AIG) in the unsatisfiability proof would guarantee a sufficient set; however, too many latch variables in the proof may lead to too many refinement candidates and a time-consuming refinement minimization procedure. Oftentimes, some latch variables can be eliminated without affecting unsatisfiability. If we define the latch-bridge-pair for a latch to be the pair of the present and the next state variables of a latch for the same time frame, we can claim that a latch can be eliminated without damaging the unsatisfiability of the formula if none of its latch-bridge-pairs appears in the proof [11]. So if we pick all latches that have at least one latch-bridge-pair in the unsatisfiability proof, we can build a sufficient set. Since each such latch works like a bridge for propagating the implications, we call this kind of abstraction extraction bridge abstraction.

After refinement candidates are generated, to keep the abstract model compact after refining, we use the structural guidance provided by relative correlation [12] to order the candidates for the unsatisfiability proof-guided refinement procedure, UNSATPROOFGUIDEDRM, the result of which is a minimal refinement set (see Sect. 3.2). This refinement will be added to the current abstract model to obtain a new abstraction. Then a refinement prediction procedure, GETREFINEMENTFROMREFPREDICT, described in Sect. 3.3, is applied to it to generate another refinement. The final new abstract model, which will be returned to the main procedure, will be obtained by adding the second refinement to the abstract model.

In the abstraction refinement approach, if abstract models become close to concrete models, due to the inner abstraction refinement mechanism of SAT solver, we may not gain much by using abstraction refinement even after various techniques like IAROSAT, URM and RP_-descibed in the sequel-have been used. So, at each length, we calculate what percentage the abstract model is in the concrete model. If it is larger than a threshold, we switch to flat model checking. We call this simple, yet effective technique abstraction switching.

\subsection{Improved AROSAT}

Given a circuit $\mathcal{C}$, let $V$ be a set of variables of $\mathcal{C}$, and $\left\{V_{1}, V_{2}, \ldots, V_{n}\right\}$ a partition of $V$. Then $V_{1}, V_{2}, \ldots, V_{n}$ are called layers of $\mathcal{C}$. AROSAT [12] is a SAT algorithm designed especially for the abstraction and refinement approach, to generate unsatisfiability proofs that use latch variables corresponding to fewer different latches. In 
AROSAT, layered constraints are enforced on the choice of decision variables and on implication propagation to make sure only necessary latch variables are involved in the SAT search. AROSAT normally obtains much better unsatisfiability proof than an ordinary SAT solver, however, the speed of AROSAT is much slower, 5 times slower according to [11]. Here we propose an improved version of the old AROSAT which is called IAROSAT. Compared to AROSAT, IAROSAT has the following features:

In AROSAT, only latch variables are divided into different layers and given higher priority to be selected as decision variables than other variables. This causes the decision variables to be just latch variables in most cases. In [6] the observation was made that the choice of branching variables should be very dynamic. Accordingly, in IAROSAT, instead of concentrating only on latch variables for decision variable selection, we divide the whole variable space into different layers, and all variables in the same layer have the same priority. We first form clusters of latches according to the latch order with respect to their relative correlation values. We then start from latches in higher priority clusters, cluster by cluster, to do cone-of-influence search. We generate the layers by collecting all variables which are in the direct cone-of-influence, DCOI for short, of latches in a certain cluster but not in the DCOI of any latch in any higher priority clusters, as the corresponding layer.

In AROSAT, latch variables are divided into different layers and implications can be propagated beyond the border of a certain layer if and only if all variables in higher priority layer have all been assigned. Hence, we have the following lemma:

Lemma 1. In AROSAT, if a variable in Layer $i$ has been assigned a value, the sub-SAT instance formed by collecting the clauses containing only variables belonging to the $i-1$ highest priority layers is satisfiable.

From Lemma11 we see that once a variable has been assigned, keeping layers at higher priority separate is meaningless because unless some variables outside these layers are involved, there is no way that we can get an unsatisfiability proof. So, in IAROSAT, if any layer is involved in the SAT searching process, we just merge this layer with all the layers with higher priority. In IAROSAT, to gain more speed, score decay is allowed, but only within each layer to guarantee the different priorities of layers. From our experiments, the speed of IAROSAT is only 2.8 times slower than a conventional SAT solver while AROSAT is 5 times slower.

\subsection{Unsatisfiability Proof-Guided Refinement Minimization (URM)}

In PureSAT [12], refinement minimization is an important technique to guarantee the final refinement is minimal so that the cumulative abstract models do not grow too quickly. However, even after we apply IAROSAT, the refinement candidates generated directly from unsatisfiability proofs may still be numerous, which results in a timeconsuming refinement minimization procedure.

Here we propose an unsatisfiability proof-guided refinement minimization procedure which works as follows: After the refinement candidates are generated, they are given to the refinement minimization engine to be tested one by one. During each test, an unsatisfiability proof is generated. Since we are trying to find a small set of latches, which, when added to the old abstraction, can form a new abstraction without any current counterexamples, any untested latches that do not appear in the current unsatisfiability proof 
can be eliminated from the candidate list. In this way, one test may eliminate multiple candidates.

\subsection{Refinement Prediction (RP)}

The refinement minimization engine is only responsible for finding a sufficient set to kill all counterexamples up to the current length without any concern about future refinements. Such an approach guarantees a minimal refinement. However, some latches which are also eliminated would prevent spurious abstract counterexamples and decrease the number of refinement iterations.

Here we propose a refinement prediction approach that utilizes current available information. The criteria we use for judging whether a latch is a good candidate for future refinements are the following:

1. Including this latch into the current abstract model will not add too much burden to the model checking engine.

2. This latch is closely related to the current abstraction.

For the first criterion, since we only add all the gates in the DCOI of a latch into our abstract model once we include this latch into the current abstraction, we tend to add those latches such that most gates of their direct cones are already in the current abstraction. For the second criterion, we have two assumptions: 1) If most gates in the DCOI of a latch are in the current abstraction, then this latch is closely related to current abstract model. 2) If we take the current abstraction as core and do a DFS and we only consider latches, then a latch with a smaller DFS level is more closely related to the current abstract model than a latch with a larger DFS level. Considering all these criteria and assumptions, we regard a latch as a future refinement and add it to current abstraction if most gates in its DCOI are already in the current abstraction and it has a very small DFS level.

\section{Implementation}

\subsection{AIG-Based Implementation}

Our implementation is based on AIG, which is a Boolean circuit containing only AND gates and inverters. To reuse the AIG nodes previously built as much as possible, when we unfold the AIG, we directly build it for the whole concrete model. Then, for each abstraction, we mark all the nodes which are in the current abstract model, and only these nodes marked are involved in the SAT solving process.

If the SAT instance turns out to be unsatisfiable and an interpolant needs to be generated, a resolution graph is then created, the leaves of which are CNF clauses translated from AIG nodes, and the root of which is the empty clause. The interpolant is then computed based on this resolution graph.

\subsection{Elimination of Pseudo-global Variables}

In the interpolation-based model checking algorithm [15], the model checking problem is first translated into a SAT instance in the form of CNF clauses, and then all clauses are 


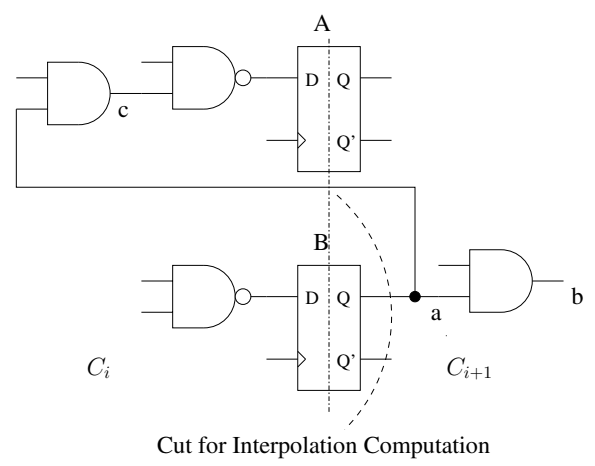

Fig. 3. Elimination of pseudo-global variables

divided into disjoint subset $\left\{C_{1}, C_{2}\right\}$, which corresponds to placing a cut in the original circuit. A variable is a global variable if it appears in both subsets; otherwise, it is a local variable. Only global variables can appear in interpolants. If an interpolant is to be an overapproximation of the reachable states, the global variables are normally latch variables. However, if interpolants are computed on abstract models, we may introduce pseudo-global variables, PGV for short.

Figure 3 illustrates this problem. In the figure, $A$ and $B$ are two latches. In the concrete model, $a$ is a global variable since it appears in both clauses of $(\neg c \vee a)$ and $(\neg c \vee b)$, which belong to $C_{1}$ and $C_{2}$, respectively. However, if we assume $A$ is in the abstract model while $B$ is not, then $a$ is treated as a pseudo-input, that is, all the logic feeding latch $B$ is eliminated. After this abstraction, $a$ remains a global variable because it still appears in $C_{1}$ and $C_{2}$, which means that $a$ may appear in the computed interpolant, while an expression in terms of only visible latch variables is desired here.

There may be various ways to deal with this problem. In this paper, we use a method called variable-splitting, which works as follows: Before the SAT instance is sent to the SAT solver, a preprocessing step finds all the possible PGVs, and splits each of them into two variables; one is connected to the nodes of $C_{i}$, the other is connected to the nodes of $C_{i+1}$. After the preprocessing step, only visible latch variables are global variables. A postprocessing step for recovering those PGVs is also utilized after the interpolant is generated.

\section{Experimental Results}

To evaluate the efficiency of our algorithm, we compared different algorithms on 21 test cases using models from both industry and the VIS verification benchmarks [3, 19]. Thirteen of the properties fail; the other 8 pass. In the following tables, failing examples are shown on top, and both kinds of examples are sorted by increasing number of latches in the concrete model. A line is used to separate the two kinds of examples. All the experiments were run under Linux on an IBM IntelliStation with a 1.7 GHz Intel Pentium 4 CPU and $2 \mathrm{~GB}$ of RAM.

Table 1 shows the comparison among five algorithms: 1) the algorithm of [12], named PureSAT, 2) the PureSAT+AROSAT algorithm [11], named AROSAT, 3) our 
Table 1. Experimental results

\begin{tabular}{|c|c|c|c|c|c|c|c|c|c|c|c|c|c|}
\hline model & size & Fail// & PureSAT & \multicolumn{2}{|c|}{ AROSAT } & \multicolumn{2}{|c|}{ AigIP } & \multicolumn{3}{|c|}{ IP } & \multicolumn{2}{|c|}{ IPAR } \\
\cline { 4 - 12 } & & Pass & time & len. & time & len. & time & len. & time & len. & time & len. \\
\hline D14-p1 & 96 & $\mathrm{~F}$ & 486 & 14 & 824 & 14 & 158 & 14 & 36 & 14 & 31 & 14 \\
28-batch & 108 & $\mathrm{~F}$ & 443 & 14 & 3723 & 14 & 155 & 14 & 51 & 14 & 36 & 14 \\
03-batch & 119 & $\mathrm{~F}$ & 300 & 32 & 736 & 32 & 157 & 32 & 72 & 32 & 97 & 32 \\
bj-2 & 122 & $\mathrm{~F}$ & 88 & 17 & 201 & 17 & 54 & 17 & 65 & 17 & 40 & 17 \\
18-batch & 133 & $\mathrm{~F}$ & 2148 & 23 & TO & $(21)$ & 1391 & 23 & 571 & 23 & 426 & 23 \\
06-batch & 140 & $\mathrm{~F}$ & 1936 & 31 & TO & $(29)$ & 1935 & 31 & 257 & 31 & 218 & 31 \\
04-batch & 252 & $\mathrm{~F}$ & 72 & 24 & 96 & 24 & 36 & 24 & 25 & 24 & 22 & 24 \\
D5-p1 & 319 & $\mathrm{~F}$ & 51 & 31 & 32 & 31 & 15 & 31 & 27 & 31 & 14 & 31 \\
D18-p1 & 506 & $\mathrm{~F}$ & $\mathrm{MO}$ & $(22)$ & 1185 & 23 & 154 & 23 & 64 & 23 & 63 & 23 \\
D20-p1 & 562 & $\mathrm{~F}$ & $\mathrm{MO}$ & $(9)$ & 3154 & 14 & 848 & 14 & 20 & 14 & 82 & 14 \\
24-batch-3 & 701 & $\mathrm{~F}$ & $\mathrm{MO}$ & $(21)$ & TO & $(12)$ & 6447 & 24 & 2425 & 24 & 1956 & 24 \\
24-batch-1 & 766 & $\mathrm{~F}$ & $\mathrm{MO}$ & $(20)$ & TO & $(12)$ & 5435 & 24 & 1133 & 24 & 2179 & 24 \\
24-batch-2 & 766 & $\mathrm{~F}$ & $\mathrm{MO}$ & $(21)$ & TO & $(12)$ & 6874 & 24 & 1760 & 24 & 2786 & 24 \\
\hline bj-3 & 122 & $\mathrm{P}$ & TO & $(48)$ & TO & $(49)$ & TO & $(16-14)$ & 7352 & $13-14$ & 507 & $6-10$ \\
bj-4 & 122 & $\mathrm{P}$ & TO & $(37)$ & TO & $(52)$ & 242 & $7-8$ & TO & $(25-2)$ & 578 & $7-12$ \\
02-batch-2 & 140 & $\mathrm{P}$ & TO & $(109)$ & TO & $(86)$ & 6.7 & $7-19$ & 1.2 & $4-12$ & 6.8 & $7-19$ \\
02-batch-1 & 141 & $\mathrm{P}$ & $\mathrm{MO}$ & $(111)$ & TO & $(84)$ & 133 & $24-24$ & 993 & $66-18$ & 134 & $24-24$ \\
25-batch & 218 & $\mathrm{P}$ & 7 & 9 & 20 & 9 & 3.8 & $6-4$ & 1.2 & $4-4$ & 3.1 & $5-3$ \\
motoro & 222 & $\mathrm{P}$ & 2100 & 14 & 1219 & 14 & 39 & $4-3$ & 70 & $3-6$ & 45 & $4-3$ \\
IU-p1 & 4494 & $\mathrm{P}$ & $\mathrm{MO}$ & $(69)$ & TO & $(19)$ & 664 & $24-17$ & TO & $(35-6)$ & 2136 & $72-8$ \\
IU-p2 & 4494 & $\mathrm{P}$ & 780 & 11 & 868 & 11 & 192 & $8-8$ & 73 & $3-6$ & 92 & $2-5$ \\
\hline
\end{tabular}

basic algorithm which combines together interpolation, abstraction by localization reduction, and refinement based on unsatisfiability proof and bridge abstraction, named AigIP, 4) the flat interpolation algorithm of [15], named IP, and 5) our final algorithm, which is built on top of AigIP by adding various techniques described in Sect. 3] called IPAR. A comparison to PureSAT and AROSAT shows that our baseline algorithm, AigIP, is competitive, and that our implementation of interpolation is efficient.

In Table 1 the first column gives the names of models, the second column shows the size of each model in terms of latches in the COI of the property. The third column tells whether the property of the model is passing or failing. In this and the following tables, $M O$ means memory out, and TO means time out where the time limit is set to 12000 s. Columns 4-13 indicate the results for the five algorithms. For each algorithm, the left column is the total time, and the right one is the termination length: for a failing property, it is the length of the shortest counterexample; for a passing property, it is the length at which the property is proved. For a passing property, in PureSAT and AROSAT, the termination length is the longest simple path +1 [17], while for AigIP, IP, and IPAR, the first number is the length on termination, the second number is the number of iterations at the length on termination. For example, for IU-p1, IPAR terminated at the 8th iteration of length 72 . Since we increase the current checking length by $L=L+$ iterations -1 , it is fair to think of termination length as the sum of length on termination and the number of iterations for this length. In this and the following tables, a number in parentheses indicates the length at which the run was aborted due to insuffi- 
cient resources. From Table 1 if we compare PureSAT, AROSAT vs. IPAR, we see that we got a substantial improvement over our starting point, which is due to two factors: one is interpolation is more efficient than simple path checking in terms of termination length and computational cost, the other is that various techniques we developed, which are described in Sect. 3, prove to be very efficient in the abstraction refinement procedure. If we compare IP and IPAR, we can see that for true properties, IPAR wins most of the time, especially in large examples. For some passing examples, like IU-p2, even though IPAR loses because of the overhead, it still wins if we consider the termination length. For false properties, IPAR is a bit better, but generally, comparable to IP. Hence, we can conclude that abstraction helps to shorten termination length.

Comparison of AigIP and IP shows that the former is significantly faster for passing properties, but correspondingly slower for failing properties. Table 2 shows that the problem is due to excessive time spent in refinement. The improvements brought by the various techniques described in Sect. 3 (except for abstraction switching) are also shown in Table 2, which compares the algorithms AigIP and IPAR3. Algorithm IPAR is not used here because the information on refinement in algorithm IPAR may not be accurate enough due to abstraction switching. Columns 4-6 and 7-9 represent data from algorithms AigIP and IPAR3, respectively. In each algorithm, we collect the data for refinement time, total time, and the percentage of total time spent for refinement. From this table, we can see that the techniques in Sect.3 drastically decrease refinement

Table 2. Impact of refinement computation on CPU time

\begin{tabular}{|c|c|c|c|c|c|c|c|c|}
\hline Model & Size & F/P & \multicolumn{3}{|c|}{ AigIP } & \multicolumn{3}{|c|}{ IPAR3 } \\
\cline { 4 - 9 } & & & Ref. & Total & Perc. & Ref. & Total & Perc. \\
\hline D14-p1 & 96 & 14 & 128 & 158 & 81 & 29 & 54 & 54 \\
28-batch & 108 & 14 & 121 & 155 & 78 & 27 & 60 & 45 \\
03-batch & 119 & 32 & 106 & 157 & 68 & 42 & 142 & 30 \\
bj-2 & 122 & 17 & 6 & 54 & 11 & 4 & 53 & 8 \\
18-batch & 133 & 23 & 920 & 1391 & 66 & 558 & 1432 & 39 \\
06-batch & 140 & 31 & 1643 & 1935 & 85 & 376 & 547 & 69 \\
04-batch & 252 & 24 & 20 & 36 & 56 & 8 & 21 & 38 \\
D5-p1 & 319 & 31 & 4 & 15 & 27 & 3 & 13 & 23 \\
D18-p1 & 506 & 23 & 120 & 154 & 78 & 93 & 128 & 73 \\
D20-p1 & 562 & 14 & 760 & 848 & 90 & 736 & 814 & 90 \\
24-batch-3 & 701 & 24 & 4930 & 6447 & 76 & 1380 & 2964 & 47 \\
24-batch-1 & 766 & 24 & 4741 & 5435 & 87 & 1375 & 2198 & 63 \\
24-batch-2 & 766 & 24 & 4455 & 6874 & 65 & 2038 & 2901 & 70 \\
\hline bj-3 & 122 & $\mathrm{P}$ & 58 & $>12000$ & $<0.5$ & 49 & 504 & 10 \\
bj-4 & 122 & $\mathrm{P}$ & 109 & 242 & 45 & 47 & 180 & 26 \\
02-batch-2 & 140 & $\mathrm{P}$ & 0.17 & 6.7 & 3 & 0.1 & 6.8 & 1 \\
02-batch-1 & 141 & $\mathrm{P}$ & 0.16 & 133 & 0.1 & 0.16 & 48 & 0.3 \\
25-batch & 218 & $\mathrm{P}$ & 1.15 & 3.8 & 30 & 0.5 & 3 & 17 \\
motoro & 222 & $\mathrm{P}$ & 12 & 39 & 31 & 17 & 44 & 38 \\
IU-p1 & 4494 & $\mathrm{P}$ & 101 & 664 & 15 & 452 & 2127 & 21 \\
IU-p2 & 4494 & $\mathrm{P}$ & 11 & 192 & 6 & 0 & 92 & 0 \\
\hline
\end{tabular}


Table 3. Impact of different speedup techniques

\begin{tabular}{|c|c|c|c|c|c|c|c|}
\hline model & size & F/P & AigIP & IPAR1 & IPAR2 & IPAR3 & IPAR \\
\hline D14-p1 & 96 & 14 & 158 & 133 & 118 & 54 & 31 \\
28-batch & 108 & 14 & 155 & 96 & 99 & 60 & 36 \\
03-batch & 119 & 32 & 157 & 168 & 144 & 142 & 97 \\
bj-2 & 122 & 17 & 54 & 40 & 38 & 53 & 40 \\
18-batch & 133 & 23 & 1391 & 1272 & 1057 & 1432 & 426 \\
06-batch & 140 & 31 & 1935 & 658 & 535 & 547 & 218 \\
04-batch & 252 & 24 & 36 & 18 & 19 & 21 & 22 \\
D5-p1 & 319 & 31 & 15 & 13 & 13 & 13 & 14 \\
D18-p1 & 506 & 23 & 154 & 126 & 127 & 128 & 63 \\
D20-p1 & 562 & 14 & 848 & 803 & 582 & 814 & 82 \\
24-batch-3 & 701 & 24 & 6447 & 5793 & 5312 & 2964 & 1956 \\
24-batch-1 & 766 & 24 & 5435 & 2046 & 1921 & 2198 & 2179 \\
24-batch-2 & 766 & 24 & 6874 & 4123 & 3954 & 2901 & 2786 \\
\hline bj-3 & 122 & $\mathrm{P}$ & TO & 502 & 498 & 504 & 507 \\
bj-4 & 122 & $\mathrm{P}$ & 242 & 575 & 545 & 180 & 578 \\
02-batch-2 & 140 & $\mathrm{P}$ & 6.7 & 3.4 & 3.4 & 6.8 & 6.8 \\
02-batch-1 & 141 & $\mathrm{P}$ & 133 & 132 & 132 & 48 & 134 \\
25-batch & 218 & $\mathrm{P}$ & 3.8 & 2.5 & 2.6 & 3.1 & 3.1 \\
motoro & 222 & $\mathrm{P}$ & 39 & 46 & 42 & 44 & 45 \\
IU-p1 & 4494 & $\mathrm{P}$ & 664 & 2232 & 2212 & 2127 & 2136 \\
IU-p2 & 4494 & $\mathrm{P}$ & 192 & 128 & 128 & 92 & 92 \\
\hline
\end{tabular}

time for failing properties. Even for examples like 24-batch-2, in which the percentage of refinement time increases, we still get a much smaller absolute refinement time. However, for passing properties, we don't see much improvement, and even occasional deterioration. The reasons are two: First, most time for passing properties is spent on convergence checking instead of refinement, while techniques we described in Sect. 3 are mainly designed to tackle the problem of time-consuming refinement. Second, the termination lengths for passing properties are not very stable.

A detailed examination of different techniques we described in Sect. 3 is shown in Table 3, which compares five variants of the IPAR algorithm. The basic one is AigIP in Table 1, the other four algorithms are formed by adding techniques one at the time: 1) IAROSAT gives IPAR1, 2) Unsatisfiability proof based refinement minimization gives IPAR2, 3) Refinement prediction results in IPAR3, and 4) Abstraction switching, which finally gives IPAR. Columns 4-8 show the results for these algorithms, respectively. For failing properties, a clear trend of improvements can be seen over these five algorithms from this table. For passing properties, we don't gain much because of the same reasons as we pointed out in the discussion for Table 2 .

\section{Conclusions}

We have presented an abstraction refinement algorithm for model checking that uses interpolants to prove termination. Experimental results show that, in most cases, abstraction helps prove termination for passing properties. The challenge of combining 
the two techniques lies in the overhead associated with the computation of refinement, which incurs a significant overhead for failing properties. We have therefore developed a set of techniques designed to speed up refinement to the point that the new algorithm is competitive for failing properties, while retaining its advantage for passing properties. Our results support the conclusion that, in spite of the abstraction implicitly performed by a modern SAT solver, there is benefit in applying an explicit abstraction refinement scheme.

\section{References}

[1] M. Awedh and F. Somenzi. Proving more properties with bounded model checking. In R. Alur and D. Peled, editors, Sixteenth Conference on Computer Aided Verification (CAV'04), pages 96-108. Springer-Verlag, Berlin, July 2004. LNCS 3114.

[2] A. Biere, A. Cimatti, E. Clarke, and Y. Zhu. Symbolic model checking without BDDs. In Fifth International Conference on Tools and Algorithms for Construction and Analysis of Systems (TACAS'99), pages 193-207, Amsterdam, The Netherlands, Mar. 1999. LNCS 1579.

[3] R. K. Brayton et al. VIS: A system for verification and synthesis. In T. Henzinger and R. Alur, editors, Eighth Conference on Computer Aided Verification (CAV'96), pages 428432. Springer-Verlag, Rutgers University, 1996. LNCS 1102.

[4] P. Chauhan, E. Clarke, J. Kukula, S. Sapra, H. Veith, and D. Wang. Automated abstraction refinement for model checking large state spaces using SAT based conflict analysis. In M. D. Aagaard and J. W. O'Leary, editors, Formal Methods in Computer Aided Design, pages 33-51. Springer-Verlag, Nov. 2002. LNCS 2517.

[5] E. Clarke, A. Gupta, J. Kukula, and O. Strichman. SAT based abstraction-refinement using ILP and machine learning. In E. Brinksma and K. G. Larsen, editors, Fourteenth Conference on Computer Aided Verification (CAV 2002), pages 265-279. Springer-Verlag, July 2002. LNCS 2404.

[6] E. Goldberg and Y. Novikov. BerkMin: A fast and robust SAT-solver. In Proceedings of the Conference on Design, Automation and Test in Europe, pages 142-149, Paris, France, Mar. 2002.

[7] E. Goldberg and Y. Novikov. Verification of proofs of unsatisfiability for CNF formulas. In Design, Automation and Test in Europe (DATE'03), pages 886-891, Munich, Germany, Mar. 2003.

[8] A. Gupta and O. Strichman. Abstraction refinement for bounded model checking. In Seventeenth Conference on Computer Aided Verification (CAV'05), pages 112-124. SpringerVerlag, Berlin, July 2005. LNCS 3576.

[9] A. Kuehlmann and F. Krohm. Equivalence checking using cuts and heaps. In Proceedings of the Design Automation Conference, pages 263-268, Anaheim, CA, June 1997.

[10] R. P. Kurshan. Computer-Aided Verification of Coordinating Processes. Princeton University Press, Princeton, NJ, 1994.

[11] B. Li and F. Somenzi. Efficient computation of small abstraction refinements. In Proceedings of the International Conference on Computer-Aided Design, pages 518-525, San Jose, CA, Nov. 2004.

[12] B. Li, C. Wang, and F. Somenzi. Abstraction refinement in symbolic model checking using satisfiability as the only decision procedure. Software Tools for Technology Transfer, 7(2):143-155, Apr. 2005.

[13] J. P. Marques-Silva. Improvements to the implementation of interpolant-based model checking. In Correct Hardware Design and Verification Methods (CHARME'05), pages 367-370, Saarbrucken, Germany, Oct. 2005. Springer-Verlag. LNCS 3725. 
[14] K. L. McMillan. Applying SAT methods in unbounded symbolic model checking. In E. Brinksma and K. G. Larsen, editors, Fourteenth Conference on Computer Aided Verification (CAV'02), pages 250-264. Springer-Verlag, Berlin, July 2002. LNCS 2404.

[15] K. L. McMillan. Interpolation and SAT-based model checking. In W. A. Hunt, Jr. and F. Somenzi, editors, Fifteenth Conference on Computer Aided Verification (CAV'03), pages 1-13. Springer-Verlag, Berlin, July 2003. LNCS 2725.

[16] K. L. McMillan and N. Amla. Automatic abstraction without counterexamples. In International Conference on Tools and Algorithms for Construction and Analysis of Systems (TACAS'03), pages 2-17, Warsaw, Poland, Apr. 2003. LNCS 2619.

[17] M. Sheeran, S. Singh, and G. Stålmarck. Checking safety properties using induction and a SAT-solver. In W. A. Hunt, Jr. and S. D. Johnson, editors, Formal Methods in Computer Aided Design, pages 108-125. Springer-Verlag, Nov. 2000. LNCS 1954.

[18] J. P. M. Silva and K. A. Sakallah. Grasp-a new search algorithm for satisfiability. In Proceedings of the International Conference on Computer-Aided Design, pages 220-227, San Jose, CA, Nov. 1996.

[19] URL: http://vlsi.colorado.edu/ vis.

[20] C. Wang, B. Li, H. Jin, G. D. Hachtel, and F. Somenzi. Improving Ariadne's bundle by following multiple threads in abstraction refinement. In Proceedings of the International Conference on Computer-Aided Design, pages 408-415, Nov. 2003.

[21] L. Zhang and S. Malik. Validating SAT solvers using an independent resolution-based checker: Practical implementations and other applications. In Design, Automation and Test in Europe (DATE'03), pages 880-885, Munich, Germany, Mar. 2003. 